\title{
A Three-Dimensional Recovery-Based Discontinuous Galerkin Method for Turbulence Simulations
}

\author{
Eric Johnsen ${ }^{*}$ Sreenivas Varadan ${ }^{\dagger}$ and Bram van Leer ${ }^{\ddagger}$ \\ University of Michigan, Ann Arbor, MI 48109, USA
}

\begin{abstract}
Discontinuous Galerkin (DG) methods have recently received much attention because of their portability to complex geometries, scalability in parallel architectures and relatively simple extension to high order. However, their implementation for compressible turbulence problems is not straightforward, e.g., due to parameter-free limiting for orders greater than first and the lack of a consistent high-order diffusion scheme for DG. To address this last point, Van Leer proposed the idea of recovery-based discontinuous Galerkin (RDG) approaches. In the present work, an explicit recovery-based diffusion scheme is developed in three dimensions on a Cartesian grid to solve incompressible and slightly compressible turbulence problems. It is shown that the selection of an optimal recovery and enhancement basis preserves the super-convergence property of this scheme (eighth order for $P=2$, where $P$ is the order of the polynomial basis) for the three-dimensional Navier-Stokes equations. Test problems confirm the efficient and accurate application of the present recovery-based discontinuous Galerkin method to incompressible and slightly compressible turbulence problems.
\end{abstract}

\section{Introduction}

Discontinuous Galerkin (DG) methods combine advantages of finite volume (FV) and finite element (FE) approaches into a single framework. The Galerkin formulation, borrowed from the FE methodology, produces a high-order accurate and compact-stencil scheme with several advantages over high-order FV and finite difference (FD) methods, such as their portability to complex geometries, scalability in parallel architecture and relatively simple extension to high order. The discontinuous nature of the basis functions in each cell introduces means for adding numerical dissipation in the convective terms, such that, unlike traditional FE schemes, discontinuous solutions can be captured in a stable fashion. The DG method was originally introduced for solving the steady-state neutron transport equation on triangular meshes, ${ }^{24}$ and later analyzed. ${ }^{16,18,23,25}$ The introduction of the Runge-Kutta DG (RKDG) method by Cockburn and $\mathrm{Shu}^{7-10}$ made this approach appealing for time-dependent convection-dominated problems. The RKDG method was shown to be well-suited to handle shocks through the use of slope limiters in one and two dimensions, on rectangular and triangular meshes. Similar to FV schemes, DG methods use Riemann solvers to determine the inter-cell flux and thus introduce the appropriate amount of dissipation at the discontinuities.

However, the main difficulty preventing a simple extension to solving diffusive terms (i.e., second-order derivatives or differences), such as in the Navier-Stokes equations, is the fact that derivatives are undefined at cell edges due to the discontinuity in the basis functions between neighboring cells. Two main approaches have been followed in the past: one in which gradient information is provided and which is stabilized by interior penalty terms or artificial diffusion with adjustable parameters; and another more recent based on the idea of recovery of the underlying function over neighboring cells. With the former, interior penalty methods have been used for elliptic and parabolic problems. ${ }^{1,6,12}$ Another class of methods treat the second-order partial differential equations as a system of first-order equations. ${ }^{2,3,11,22}$ Such methods have been used to solve for diffusive terms, but there is no consistent methodology and adjustable parameters are required. In recovery, ${ }^{20,21,30-32}$ The discontinuity at the interface is removed in the weak sense by a local polynomial

\footnotetext{
*Assistant Professor, Mechanical Engineering Department, AIAA Member.

${ }^{\dagger}$ Research Assistant, Mechanical Engineering Department, AIAA Member.

${ }^{\ddagger}$ Professor, Aerospace Engineering Department, AIAA Fellow.
} 
required to satisfy moments with the original solution in the elements that span the interface. The smooth recovered function is used to compute the necessary function values and derivatives at the interface. The resulting recovery-based discontinuous Galerkin method (RDG) is shown to be stable and have an accuracy of at least $2 P+2$ in one dimension, where $P$ is the order of the polynomial in each cell. The method does not require additional stabilizing terms and computes a unique value of the function derivative at the interface, which depends only on the neighboring elements. The scheme has been extended to handle nonlinear systems of equations through a solution enhancement that increases the local order of the solution by using the recovered information. The enhanced information is further used to improve the order of the recovered function in the transverse direction. Through this enhanced recovery process, the order of accuracy of diffusive terms in two-dimensional nonlinear systems of equations, such as the Navier-Stokes equations, is as good as for the one-dimensional linear diffusion equation. A similar approach has been followed for two-dimensional unstructured grids. ${ }^{4}$

The extension of RDG to three dimensions is not straightforward. We show here that it is not possible to achieve a full tensor product recovery basis after enhacement in the tangential Cartesian directions in three dimensions. Instead, an incomplete basis is formulated, which is shown to preserve the same order of accuracy as in one dimension, up to $P=2$ polynomials. The performance of the resulting enhanced and recovered DG scheme is assessed through numerical experiments for smooth problems, including incompressible and slightly compressible (i.e., no shocks) broadband turbulence.

\section{Governing equations}

The governing equations are the three-dimensional compressible Navier-Stokes equations:

$$
\frac{\partial \mathbf{Q}}{\partial t}+\nabla \cdot \mathbf{F}_{e}(\mathbf{Q})-\nabla \cdot \mathbf{F}_{v}(\mathbf{Q}, \nabla \mathbf{Q})=\mathbf{0}
$$

where the vectors of conservative variables, convective flux and viscous flux are

$$
\begin{aligned}
\mathbf{Q} & =(\rho, \rho \mathbf{u}, E)^{T}, \\
\mathbf{F}_{e}(\mathbf{Q}) & =(\rho \mathbf{u}, \rho \mathbf{u} \otimes \mathbf{u}+p \mathbf{I}, \mathbf{u}(E+p))^{T}, \\
\mathbf{F}_{v}(\mathbf{Q}, \nabla \mathbf{Q}) & =(0, \boldsymbol{\tau}, \mathbf{u} \cdot \boldsymbol{\tau}+\mathbf{q})^{T} .
\end{aligned}
$$

Here, $\rho$ is the density, $p$ is the pressure, $\mathbf{u}$ is the velocity vector, $E=\rho e+\rho \mathbf{u} \cdot \mathbf{u} / 2$ is the total energy, $e$ is the internal energy and $\mathbf{I}$ is the identity tensor. Since the fluid is assumed newtonian and making use of Stokes' assumption, the components of the viscous stress tensor $\tau$ are given by

$$
\tau_{i j}=\mu\left[\left(\frac{\partial u_{i}}{\partial x_{j}}+\frac{\partial u_{j}}{\partial x_{i}}\right)-\frac{2}{3} \delta_{i j} \frac{\partial u_{k}}{\partial x_{k}}\right]
$$

where $\mu$ is the viscosity, which follows a power law in terms of its dependence on temperature:

$$
\mu=\mu_{\text {ref }}\left(\frac{T}{T_{r e f}}\right)^{3 / 4}
$$

where $T$ is the temperature and $\mu_{r e f}$ and $T_{r e f}$ are reference values. The heat flux $\mathbf{q}$ is given by Fourier's law

$$
\mathbf{q}=-k \nabla T,
$$

where $k$ is the thermal conductivity. The fluid is assumed to be a calorically perfect gas

$$
p=\rho R T=(\gamma-1) \rho e,
$$

where $R$ is the gas constant and $\gamma$ is the ratio of specific heats. In the present calculations, the following values are used for the constants: $R=287 \mathrm{~J} / \mathrm{kgK}, \gamma=1.4$ and the Prandtl number $\operatorname{Pr}=\mu c_{p} / k=0.71$, where $c_{p}$ is the specific heats at constant pressure. 


\section{Numerical methodology}

\section{A. Galerkin formulation}

Let $D \subset \mathbb{R}^{\mathrm{n}}$ be a domain spatially discretized into $N_{e}$ non-overlapping elements $\Omega_{e}$ with associated spaces of discontinuous functions $V$ and $W$ such that

$$
D=\sum_{i=1}^{N_{e}} \Omega_{i}, \quad V=\left\{v \in V: v \in P^{k}\right\}, \quad W=\left\{\phi \in W: \phi \in P^{k}\right\},
$$

where $P^{k}$ is the space of polynomials of at most degree $k$. In the current work, Cartesian hexahedral elements are used in three dimensions. In the standard Galerkin approach, the test function space $W$ is taken to be the same as the solution space $V$.

Multiplying the system of equations 1 by a piece-wise smooth function $\phi$ and integrating over the volume of an element, one obtains the weak formulation of the equations in each $\Omega_{e}$. After integration by parts, this becomes

$$
\int_{\Omega_{e}} \partial_{t} \mathbf{Q}_{h} \phi d x+\sum_{e \in \partial \Omega_{e}} \int_{e}\left(\mathbf{F}_{e h}-\mathbf{F}_{v h}\right) \cdot \mathbf{n}_{e, \Omega_{e}} \phi d \Gamma+\int_{\Omega_{e}}\left(\mathbf{F}_{e h}-\mathbf{F}_{v h}\right) \cdot \nabla \phi d x=\mathbf{0}, \quad \forall \phi \in W,
$$

where the subscript $h$ denotes Galerkin approximations and $\mathbf{n}_{e, \Omega_{e}}$ is the unit normal along a given face. From here on, the boldface denoting vectors and tensors is dropped for simplicity. To compute the inviscid fluxes $\mathbf{F}_{e h}$, upwinding is required. For this purpose, the Roe approximate Riemann solver ${ }^{26}$ is used with a transonic entropy fix. ${ }^{13}$ The viscous fluxes $\mathbf{F}_{v h}$ are computed using recovery. ${ }^{30}$ The boundary terms are evaluated using a Gaussian quadrature.

Scaled Legendre polynomials are chosen as basis functions, e.g., $v_{i}^{k}$ is the $k^{\text {th }}$ degree solution basis function in element $i$. In multiple dimensions, a tensor product of the basis functions in each Cartesian direction is used to form the solution basis. The tensor basis is chosen such that the same high order is achieved in multiple dimensions as in one dimension. The solution in each element is expanded as

$$
Q_{h}^{i}=\sum_{k} a_{i}^{k}(t) v_{i}^{k}
$$

where $a_{i}^{k}(t)$ is the coefficient of the $k^{\text {th }}$ degree basis function in element $i$. Elements up to $P=3$ in one dimension and up to $P=2$ in three dimensions are considered in the present work. Eq. 8 can be written in semi-discrete form as

$$
\frac{d Q_{h}}{d t}=\mathbf{M}^{-1} \mathbf{R}\left(Q_{h}\right),
$$

where $\mathbf{M}$ is the (diagonal) mass matrix and $\mathbf{R}$ is a nonlinear difference operator.

\section{B. Time marching}

The system of ordinary differential equations 10 is solved using explicit Runge-Kutta schemes. The order of the time marching is chosen to be as close as possible to the order of the spatial discretization, while still being efficient. The accuracy for the convective terms is generally $K+1$ for a $K^{\text {th }}$ degree polynomial basis; it will be shown that for diffusion using recovery the order is at least $(2 K+2)$, even in three dimensions. Thus, for $K=1$, the third-order scheme ${ }^{27}$ is used, while the standard fourth-order Runge-Kutta scheme is used for $K>1$. Although not used here, time-marching schemes specifically designed for broadband problems may improve the performance. ${ }^{14}$

\section{Enhanced recovery for the viscous fluxes}

\section{Recovery}

The recovery procedure is described here for a Cartesian grid with regular hexahedral elements. Let $\Omega_{i, j, k}$ and $\Omega_{i+1, j, k}$ be adjacent elements sharing an interface $S_{i+1 / 2, j, k}$, and let $\left(x_{i}, y_{j}, z_{k}\right)$ be the location of the 
center of element $\Omega_{i, j, k}$ with $x_{i+1 / 2}, y_{j+1 / 2}, z_{k+1 / 2}$ being the $x, y, z$ locations of the respective boundaries. The following local coordinates are used in each element $\Omega_{i, j, k}$

$$
\xi=\frac{x-x_{i}}{2 / \Delta x}, \quad \eta=\frac{y-y_{j}}{2 / \Delta y}, \quad \zeta=\frac{z-z_{k}}{2 / \Delta z},
$$

and interface $S_{i+1 / 2, j, k}$

$$
\begin{array}{lll}
r=\frac{\xi-1}{2}, & s=\eta, \quad t=\zeta, & {[x, y, z] \in \Omega_{i, j, k},} \\
r=\frac{\xi+1}{2}, & s=\eta, \quad t=\zeta, & {[x, y, z] \in \Omega_{i+1, j, k},}
\end{array}
$$

where $r$ is the $x$-coordinate of $\Omega_{i, j, k} \cup \Omega_{i+1, j, k}$ and $s$ and $t$ the $y$ - and $z$-coordinates of $\Omega_{i, j, k} \cap \Omega_{i+1, j, k}$, respectively, with $[r, s, t] \in[-1,1]$.

The recovered function $\hat{F}(r, s, t)$ is obtained by matching its moments with those of the approximate solution $Q_{h}$ over both of the adjacent cells:

$$
\begin{aligned}
\iiint_{\Omega_{i, j, k}} Q_{h} v_{i, j, k}^{m} d x d y d z & =\iiint_{\Omega_{i, j, k}} \hat{F} v_{i, j, k}^{m} d x d y d z \\
\iiint_{\Omega_{i+1, j, k}} Q_{h} v_{i+1, j, k}^{m} d x d y d z & =\iiint_{\Omega_{i+1, j, k}} \hat{F} v_{i+1, j, k}^{m} d x d y d z, \quad m=1,2, \ldots,(K+1)^{3} .
\end{aligned}
$$

Thus, a linear system is obtained, which can be solved to obtain the new coefficients.

The extension to multiple dimensions is not trivial. The choice of a basis for the recovered function plays a critical role in the recovery procedure. Simple monomials can be used for the one-dimensional case, which led to a recovered function of degree $2 K+1$ and was extended to two dimensions. ${ }^{21,31}$ It was shown that recovery had to be directional to ensure that the recovery matrix is full-ranked. In other words, this means that an $x$-recovery only produces a polynomial of degree $2 K+1$ in $r$, with the degree being a mere $K$ in the $s$ and $t$ directions.

\section{Solution enhancement}

The concept of interior solution enhancement was introduced for improving both the face-tangential order of the recovered function and the order of the original solution in the volume integral of Eqs. $13 .{ }^{20}$ This procedure is important because the presence of derivatives in the viscous fluxes reduces the degree of the original polynomial and thus the accuracy of the volume integral. The enhanced solution $\hat{Q}_{h}$ in each element is determined by requiring it to satisfy moments with the initial recovered function at the cell edges. In the $x$-direction, one obtains:

$$
\begin{gathered}
\iiint_{\Omega_{i, j, k}} \hat{Q}_{h} v_{i, j, k}^{m} d x d y d z=\iiint_{\Omega_{i, j, k}} Q_{h} v_{i, j, k}^{m} d x d y d z, \quad m=1,2, \ldots,(K+1)^{3}, \\
\left.\int_{y_{j-1 / 2}}^{y_{j+1 / 2}} \int_{z_{k-1 / 2}}^{z_{k+1 / 2}} \hat{Q}_{h} v_{i, j, k}^{m}\right|_{x_{i \pm 1 / 2}} d z d y=\left.\int_{y_{j-1 / 2}}^{y_{j+1 / 2}} \int_{z_{k-1 / 2}}^{z_{k+1 / 2}} \hat{F} v_{i, j, k}^{m}\right|_{x_{i \pm 1 / 2}} d z d y, \quad m=1,2, \ldots,(K+1)^{2} .
\end{gathered}
$$

Eq. 14 states that the enhanced solution shares all the moments with the original solution, i.e., the coefficients of the original solution are unaltered. Eq. 15 determines the $2(K+1)^{2}$ higher-order terms in the $x$-direction. The solution order in $y$ and $z$ is similary enhanced by sharing moments with the $y$ and $z$ recovered functions $\hat{G}$ and $\hat{H}$ along the $y$ and $z$ edges, respectively. The $x$-, $y$ - and $z$-enhanced solution then replaces the original one in the volume integral for the viscous flux in Eqs. 2.

\section{Enhanced recovery}

The enhanced solution is subsequently used to perform an enhanced recovery in order to increase the degree of the recovered function in the face-tangential directions. Both of these steps are necessary for a truly multi-dimensional extension of the original one-dimensional recovery scheme. ${ }^{30}$ 
The following procedure is carried out: the $y$ - and $z$-enhanced solution is used for the $x$-recovery. This technique, known as Cartesian optimization, is required to maintain a compact stencil. The equations for enhanced $x$-recovery are

$$
\begin{aligned}
\iiint_{\Omega_{i, j, k}} \hat{Q}_{h, y z} v_{i, j, k}^{m} d x d y d z & =\iiint_{\Omega_{i, j, k}} \hat{F}_{e n} v_{i, j, k}^{m} d x d y d z \\
\iiint_{\Omega_{i+1, j, k}} \hat{Q}_{h, y z} v_{i+1, j, k}^{m} d x d y d z & =\iiint_{\Omega_{i+1, j, k}} \hat{F}_{e n} v_{i+1, j, k}^{m} d x d y d z, \quad m=1,2, \ldots,(K+1)^{3}+4(K+1)^{2}
\end{aligned}
$$

where $\hat{F}_{e n}(r, s, t)$ is the enhanced recovered function in the $x$-direction, $\hat{Q}_{h, y z}$ is the solution enhanced only in the $y$ and $z$ directions. A similar procedure produces $\hat{G}_{e n}(r, s, t), \hat{H}_{e n}(r, s, t)$, which are the enhanced recovered functions in the $y$ and $z$ directions associated with the $y$ and $z$ boundaries of each element. This procedure is followed to recover a smooth function for the conservative variables. From this smooth function, the point values and derivatives at the cell interfaces, necessary to compute the viscous fluxes, can be calculated.

While a three-dimensional extension of recovery is in principle similar to the two-dimensional version, ${ }^{20}$ the means to obtaining the resulting basis for the enhanced recovered functions is different in practice. Specifically, a judicious choice of tensor product coefficients $b_{i j k}$ for the original solution guarantees a tensor product basis for the initial recovery:

$$
\hat{F}(r, s, t)=\sum_{i=0}^{2 K+1} \sum_{j=0}^{K} \sum_{k=0}^{K} b_{i j k} r^{i} s^{j} t^{k}
$$

The use of interior solution enhancement increases the order of the original solution in each direction. Here, only a tangential enhancement is used for the enhanced recovery while the full interior enhanced solution is used for computing the volume integral for the viscous flux. The basis for full solution enhancement is as follows:

$$
\begin{aligned}
\hat{Q}_{h, x y z} & =\sum_{i=0}^{K} \sum_{j=0}^{K} \sum_{k=0}^{K} a_{i j k} \bar{X}^{i} \bar{Y}^{j} \bar{Z}^{k}+\sum_{i=K+1}^{K+2} \sum_{j=0}^{K} \sum_{k=0}^{K} \hat{a}_{i j k} \bar{X}^{i} \bar{Y}^{j} \bar{Z}^{k}+\sum_{i=0}^{K} \sum_{j=K+1}^{K+2} \sum_{k=0}^{K} \hat{a}_{i j k} \bar{X}^{i} \bar{Y}^{j} \bar{Z}^{k} \\
& +\sum_{i=0}^{K} \sum_{j=0}^{K} \sum_{k=K+1}^{K+2} \hat{a}_{i j k} \bar{X}^{i} \bar{Y}^{j} \bar{Z}^{k},
\end{aligned}
$$

where $\bar{X}^{i}, \bar{Y}^{j}$ and $\bar{Z}^{k}$ represent the Legendre polynomial in $x, y$ and $z$ of degree $i, j$ and $k$, respectively. Clearly, the enhancements in $x, y$ and $z$ are decoupled. The result of this Cartesian decoupling is that the enhanced recovery procedure does not produce a full tensor set, similar to what happens in two dimensions. ${ }^{20}$ Instead, an incomplete basis is obtained, e.g., in the $x$-direction:

$$
\hat{F}_{e n}(r, s, t)=\sum_{i=0}^{2 K+1} \sum_{j=0}^{K} \sum_{k=0}^{K} \bar{b}_{i j k} r^{i} s^{j} t^{k}+\sum_{i=0}^{2 K+1} \sum_{j=K+1}^{K+2} \sum_{k=0}^{K} \bar{b}_{i j k} r^{i} s^{j} t^{k}+\sum_{i=0}^{2 K+1} \sum_{j=0}^{K} \sum_{k=K+1}^{K+2} \bar{b}_{i j k} r^{i} s^{j} t^{k},
$$

where $\bar{b}_{i j k}$ represents the coefficients of the basis for the enhanced recovered function. The basis in Eq. 19 lacks high order cross-terms in $y$ and $z$ because the enhancements in $y$ and $z$ being decoupled. However, it is shown through examples that this incomplete basis does not diminish the order of accuracy in three dimensions, up to $K=2$.

In the current work, it is shown that this monomial basis for recovery results in acceptable conditions numbers up to $K=2$. For $K>2$, the condition numbers become large, such that numerical errors in the matrix inversion become significant. 
Table 1. $L_{2}$ errors in the cell average of total energy of the manufactured solution problem at $t=1$ for $P=2$. The convergence rate is approximately eigth-order.

\begin{tabular}{ccc} 
grid size & $L_{2}$ errors & $L_{2}$ order \\
\hline $10^{3}$ & $2.989 \mathrm{e}-9$ & - \\
$20^{3}$ & $1.287 \mathrm{e}-11$ & 7.8593 \\
$26^{3}$ & $1.618 \mathrm{e}-12$ & 7.9043
\end{tabular}

\section{Numerical results}

\section{A. Manufactured solution}

A three-dimensional implementation of a previous two-dimensional test case ${ }^{21}$ is employed to verify the convergence properties of the diffusion scheme. The following manufactured solution is used:

$$
\begin{aligned}
\rho & =2, \\
\rho u & =\rho v=\rho w=e^{-t} \sin (2 \pi x) \sin (2 \pi y) \sin (2 \pi z), \\
E & =5+e^{-t} \sin (2 \pi x) \sin (2 \pi y) \sin (2 \pi z),
\end{aligned}
$$

where $[x, y, z] \in[0,1]$ and $t \in[0,1]$. The boundary conditions are periodic in $x, y$,and $z$. The threedimensional Navier-Stokes equations are solved by setting the convective terms to zero and adding appropriate source terms to each equation to make Eqs. 20 the exact solution. A constant viscosity coefficient of 0.1 and a Von Neumann number (VNN) of 0.05 for $P=2$ is used. The choice of VNN, although well above the stability limit for the RDG scheme, prevents the temporal errors from dominating over spatial errors and hence affecting the convergence rate.

The convergence of the scheme is shown in Table 1 for $P=2$. Even though the enhanced recovery basis is not a complete tensor set, one obtains the same order for a three-dimensional nonlinear system of equations as for the one-dimensional linear diffusion equation ${ }^{30}$ using the RDG method, namely eighth order for $P=2$. The size of the finest grid, $26^{3}$, is chosen such that errors at the round-off level do not contaminate the results. Although not shown here for conciseness, fourth order is obtained for $P=1$, as expected.

\section{B. Turbulence}

\section{Taylor-Green vortex}

The viscous Taylor-Green vortex ${ }^{28}$ is a problem in which, from a well-resolved initial condition, the flow transitions to turbulence by three-dimensional vortex stretching, thus the generating small scales. The three-dimensional compressible Navier-Stokes equations are solved using the present RDG method. The initial conditions are given by:

$$
\begin{aligned}
u & =V_{o} \sin \left(\frac{x}{L}\right) \cos \left(\frac{y}{L}\right) \cos \left(\frac{z}{L}\right), \\
v & =-V_{o} \cos \left(\frac{x}{L}\right) \sin \left(\frac{y}{L}\right) \cos \left(\frac{z}{L}\right), \\
w & =0, \\
p & =p_{o}+\frac{\rho_{o} V_{o}^{2}}{16}\left[\cos \left(\frac{2 x}{L}\right)+\cos \left(\frac{2 y}{L}\right)\right]\left[\cos \left(\frac{2 z}{L}\right)+2\right] .
\end{aligned}
$$

The Mach number is small enough such that the solution is very close to the incompressible solution: $M_{o}=V_{o} / c_{o}=0.10$. The initial temperature is uniform. The Reynolds number for this flow is $R e=$ $\rho_{o} V_{o} L / \mu=1600$. The computational domain is $-\pi L \leq x, y, z \leq \pi L$ and the boundary conditions are all periodic. Time is non-dimensionalized by $t_{c}=L / V_{o}$. These initial conditions were those specified in the 1st International Workshop on High Order CFD Methods (January 7-8, 2012), as part of the 50th AIAA Aerospace Sciences Meeting, for which a reference solution was provided using a finite difference code.

The mean kinetic energy and enstrophy are plotted in Fig. 1 for $P=2$ and the following grids: $32^{3}$, $64^{3}$ and $96^{3}$. Small scales are generated by vortex stretching from the beginning, achieving the highest 


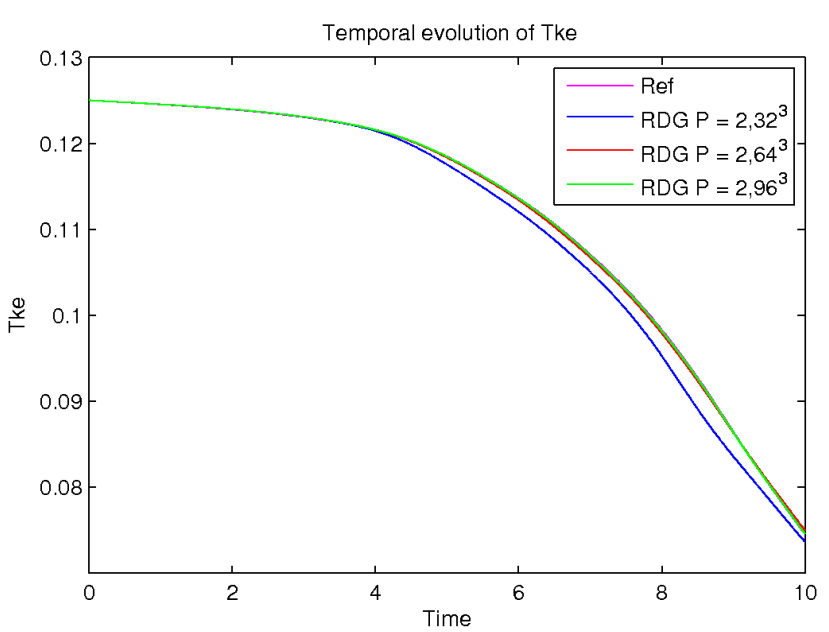

(a) Kinetic energy.

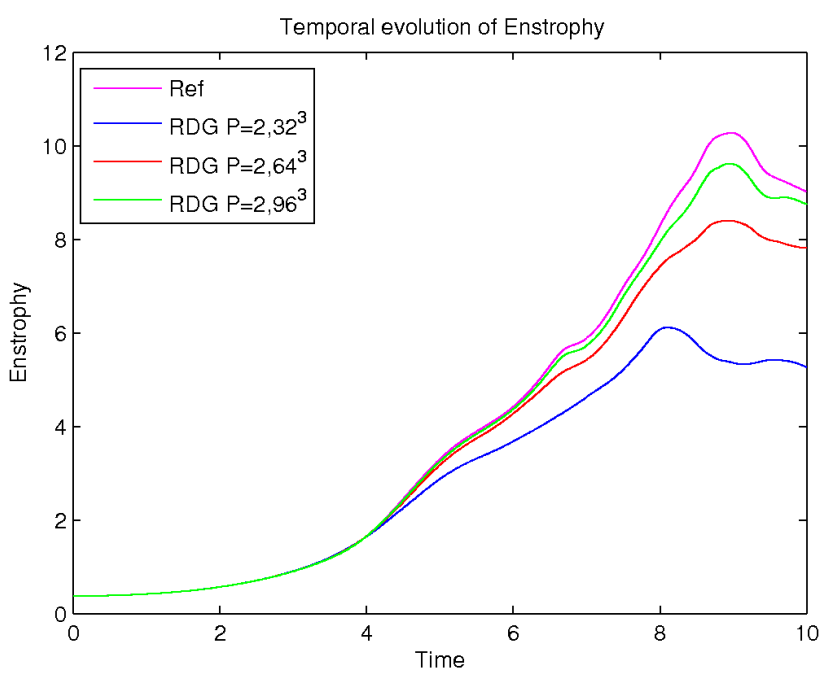

(b) Enstrophy.

Figure 1. Temporal evolution for the Taylor-Green vortex for $P=2$. Convergence is not quite achieved on the current grids at this Reynolds number.

turbulence intensity at $t \approx 9$, after which decay occurs. For the total kinetic energy, the solution on $64^{3}$ already lies essentially on top of the reference. In the enstrophy plots, the results agree with semi-analytical results ${ }^{5}$ at early times. Beyond that time, enstrophy is not quite converged, even on $96^{3}$.

\section{Compressible isotropic turbulence}

The problem of decaying compressible isotropic turbulence ${ }^{17}$ is adapted here to evaluate the performance of the present RDG method. In that problem, the turbulent Mach number $M_{t}$ is such that weak shocks (eddy shocklets), produced by the turbulent motions, form spontaneously. For the present purposes, slightly compressible turbulence $\left(M_{t}=0.2\right)$ is considered, such that shocklets do not form. Thus, no limiting is required since the solution is sufficiently smooth. This problem tests resolution properties of the present RDG method, i.e., the capability to handle broadband turbulent motions. It is noted that $M_{t}=0.3$ leads to the formation of shocklets only early in time. ${ }^{15}$

The initial conditions consist of initially constant pressure and density fields, with a random solenoidal velocity field with the following statistical description (in wavenumber space):

$$
E(k) \sim k^{4} \exp \left[-2\left(\frac{k}{k_{0}}\right)^{2}\right],
$$

where $k_{0}=4$ is taken to be the most energetic wavenumber. The initial root-mean-square velocity $u_{r m s, 0}$ is given by

$$
\frac{3 u_{r m s, 0}^{2}}{2}=\frac{1}{2}\left\langle\mathbf{u}_{0} \cdot \mathbf{u}_{\mathbf{0}}\right\rangle=\int_{0}^{\infty} E(k) d k,
$$

such that turbulent Mach number and Taylor-scale Reynolds numbers are given by:

$$
\begin{aligned}
M_{t} & =\sqrt{\frac{\langle\mathbf{u} \cdot \mathbf{u}\rangle}{3}}, \\
R e_{\lambda} & =\frac{\langle\rho\rangle u_{r m s} \lambda}{\langle\mu\rangle},
\end{aligned}
$$

where $\lambda$ is the Taylor microscale. Initially, $\lambda_{0}=2 / k_{0}$, and $R e_{\lambda, 0}=100$. Periodic boundary conditions are used on $0 \leq x, y, z \leq 2 \pi$, and time is non-dimensionalized by the eddy turn-over time, $\lambda_{0} / u_{r m s, 0}$.

The temporal evolution of the mean turbulent kinetic energy, enstrophy and dilatation is shown in Fig. 2 for using $P=2$ on the following grids: $32^{3}, 64^{3}, 96^{3}$ and $128^{3}$. Initially, because the initial conditions are not 


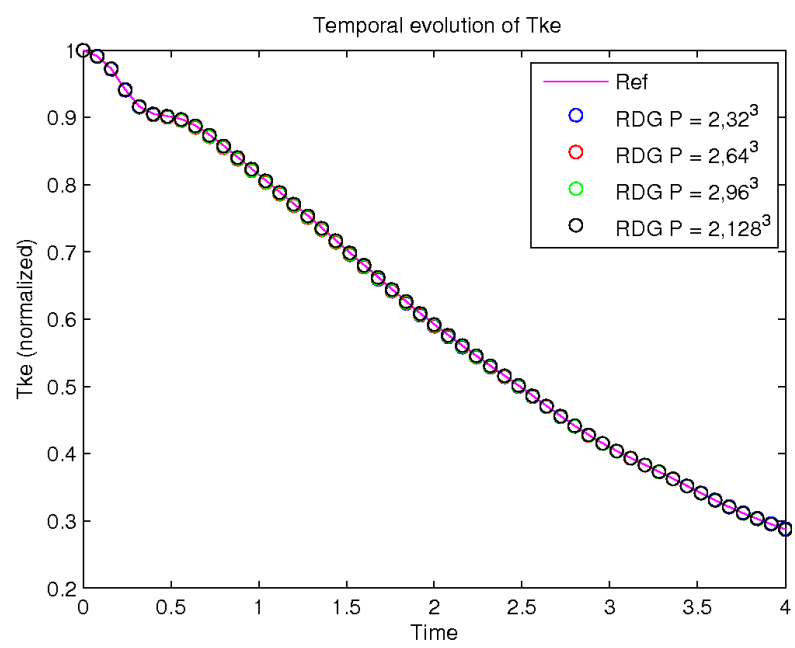

(a) Kinetic energy.

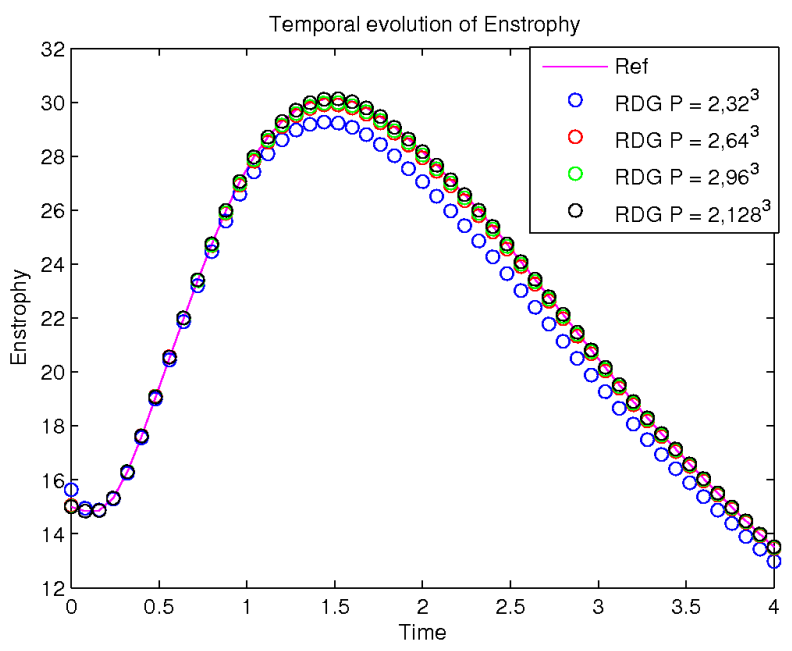

(b) Enstrophy.

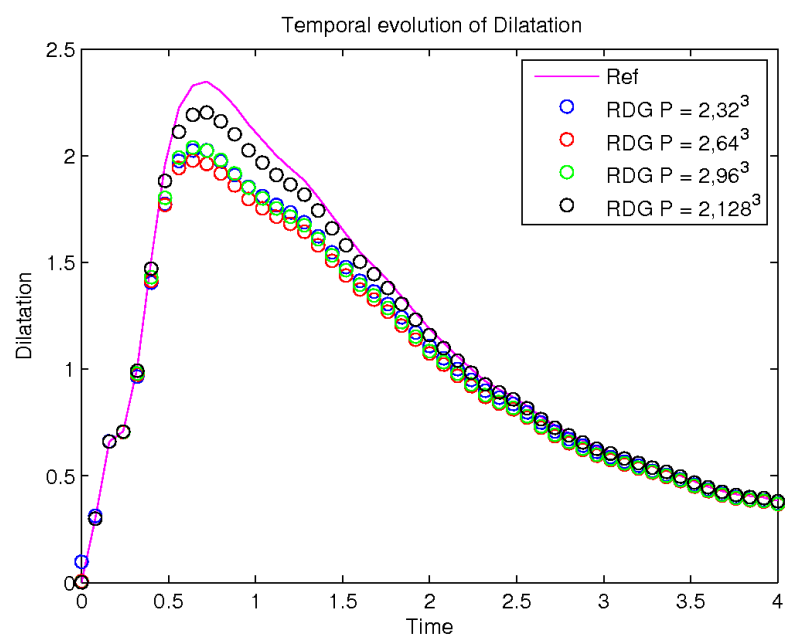

(c) Dilatation.

Figure 2. Temporal evolution in the compressible isotropic turbulence problem $\left(M_{t}=0.2, R e_{\lambda}=100\right)$ using $P=2$. Although kinetic energy and enstrophy show a converged behavior, the dilatation does not on the current grids.

in equilibrium, acoustic and entropy waves are produced and interact with the randomly distributed vorticity waves to transition to a fully-developed turbulent regime, after which the turbulence decays. For $M_{t, 0}=0.2$, shocklets never develop, such that limiting (e.g., hierarchical reconstruction) needs not be applied. As for the Taylor-Green vortex, the turbulent kinetic energy is well captured, even on the coarsest grid. The enstrophy appears to match the reference solution on $96^{3}$. However, the dilatation remains underpredicted, even with $128^{3}$ points. It should be noted that dilatation is generally a more challenging quantity to represent accurately. ${ }^{15}$

\section{Conclusions}

In the present work, the recovery procedure proposed by Van Leer ${ }^{30}$ has been extended to three dimensions to simulate broadband turbulent flows explicitly. It is shown that it is not possible to achieve a full tensor product recovery basis after enhacement in the tangential Cartesian directions in three dimensions. Instead, an incomplete basis is formulated, which is shown to preserve the same order of accuracy as in one dimension, up to $P=2$ polynomials. The selection of an optimal recovery and enhancement basis preserves the super-convergence property for smooth problems with the three-dimensional Navier-Stokes equations. 
Several moderately compressible turbulence problems (Taylor-Green vortex, decaying isotropic turbulence) are considered to evaluate the performance and resolution properties of the RDG method for broadband turbulence. Although a fully converged solution is not quite achieved, the results indicate that the present approach is viable for direct numerical simulation (DNS).

The next logical step for this work is to extend the formulation to compressible turbulence with shocks. For this purpose, some form of limiting is required. However, a significant challenge is lies in the fact that the introduction of numerical dissipation artificially damps the small turbulent scales in compressible turbulence problems. ${ }^{15}$ Preliminary compressible turbulence results will be presented and the approach based on solution-adaptive hierarchical limiting will be discussed at the meeting.

The development of recovery-based DG methods for (compressible) turbulence has far-reaching implications. High-order RDG methods are ideally suited to complex geometries and non-Cartesian grids, because of their accuracy and locality, compared to high-order finite difference methods, which lose their accuracy on non-uniform grids, and finite volume schemes, which become computationally expensive and non-trivial to implement on unstructured grids. Thus, the present work makes using high-order methods on practical engineering problems more approachable, whether for DNS or explicit large-eddy simulation (LES), for both incompressible and compressible turbulence, even on non-Cartesian grids.

\section{Acknowledgments}

This research was supported in part by the DOE NNSA/ASC under the Predictive Science Academic Alliance Program by Grant No. DEFC52-08NA28616. Some of the present calculations were run using a start-up allocation from the Extreme Science and Engineering Discovery Environment (XSEDE), which is supported by National Science Foundation grant number OCI-1053575.

\section{References}

${ }^{1}$ D. N. Arnold, F. Brezzi, B. Cockburn and L. D. Marini, Unified analysis of discontinuous Galerkin methods for elliptic problems. SIAM J. Numer. Anal. 39 (2002) 1749-1779.

${ }^{2}$ F. Bassi, S. Rebay, A high-order accurate discontinuous finite element method for the numerical solution of the compressible Navier-Stokes equations, J. Comput. Phys., 131 (1997) 267-279.

${ }^{3}$ F. Bassi, S. Rebay, G. Mariotti, S. Pedinotti, M. Savini, A high-order accurate discontinuous finite element method for viscous and turbomachinery flows, in: Proceedings of the 2nd European Conference on turbomachinery Fluid Dynamics and Thermodynamics, Belgium 1997, 99-108.

${ }^{4} \mathrm{M}$. Borrel and J. Ryan, The elastoplast discontinuous Galerkin (EDG) method for the Navier-Stokes equations, J. Comp. Phys., 231 (2012) 1-22.

${ }^{5}$ M. E. Brachet, D. I. Meiron, S. A. Orszag, B. G. Nickel, R. H. Morf and U. Frisch, Small-scale structure of the TaylorGreen vortex, J. Fluid Mech. 130 (1983) 411-452.

${ }^{6}$ F. Brezzi, G. Manzini, D. Marini, P. Pietra, A. Russo, Discontinuous Galerkin approximations for elliptic problems, Numer. Meth. Part. D. E., 16 (2000), 365-378.

${ }^{7}$ B. Cockburn and C.W. Shu, TVB Runge-Kutta local projection discontinuous Galerkin finite element method for scalar conservation laws II: General framework, Math. Comp. 52 (1989) 411-435.

${ }^{8}$ B. Cockburn, S. Y. Lin, and C. W. Shu, TVB Runge-Kutta local projection discontinuous Galerkin finite element method for conservation laws III: One dimensional systems, J. Comput. Phys. 84, 90 (1989).

${ }^{9}$ B. Cockburn, S. Hou and C. W. Shu, TVB Runge-Kutta local projection discontinuous Galerkin finite element method for conservation laws IV: One dimensional systems: The multidimensional case, Math. Comp. 54 (1990) 545-581.

${ }^{10}$ B. Cockburn and C. W. Shu, The Runge-Kutta discontinuous Galerkin method for conservation laws V: Multidimensional systems, J. Comput. Phys. 141 (1998) 199-224.

${ }^{11}$ B. Cockburn, C. W. Shu, Runge-Kutta discontinuous Galerkin methods for time-dependent convection-diffusion systems, SIAM J. Numer. Anal., 35 (1998) 2440-2463.

12 J. Douglas and T. Dupont, Interior penalty procedures for elliptic and parabolic Galerkin methods, Lect. Notes Phys. 58 (1976) 207-216.

${ }^{13}$ A. Harten and J. M. Hyman, Self-adjusting grid methods for one-dimensional hyperbolic conservation laws, J. Comput. Phys. 50 (1983) 235-269.

${ }^{14}$ F. Q. Hu, M. Y. Hussaini and J. L. Manthey, Manthey, Low-dissipation and low-dispersion Runge-Kutta schemes for computational acoustics, J. Comput. Phys. 124 (1996) 177-191.

${ }^{15}$ E. Johnsen, J. Larsson, A. V. Bhagatwala, W. H. Cabot, P. Moin, B. J. Olson, P. S. Rawat, S. K. Shankar, B. Sjogreen, H. C. Yee, X. Zhong and S. K. Lele, Assessment of high resolution methods for numerical simulations of compressible turbulence, J. Comput. Phys. 228 (2010) 1213-1237.

${ }^{16}$ C. Johnson and J. Pitkaranta, An analysis of the discontinuous Galerkin method for a scalar hyperbolic equation, Math. Comp. 46 (1986) 1-26.

${ }^{17}$ S. Lee, S. K. Lele and P. Moin, Eddy shocklets in decaying compressible turbulence, Phys. Fluids A 3 (1991) 657-664. 
${ }^{18} \mathrm{P}$. LeSaint and P. A. Raviart, On a finite element method for solving the neutron transport equation, Mathematical aspects of finite elements in partial differential equations (C. de Boor, Ed.), Academic Press, 89 (1974).

${ }^{19}$ K. H. M. Lo, A space-time Discontinuous Galerkin Method for Navier-Stokes with recovery, Ph.D. thesis, University of Michigan.

${ }^{20} \mathrm{M}$. Lo and B. van Leer, Analysis and Implementation of Recovery-Based Discontinuous Galerkin for Diffusion, AIAA paper (2009) 2009-3786.

${ }^{21}$ M. Lo and B. van Leer, Recovery-based discontinuous Galerkin for Navier-Stokes viscous terms, AIAA paper (2011) 2011-3406.

22 J. Peraire, P. O. Persson The compact discontinuous Galerkin (CDG) method for elliptic problems, SIAM J. Sci. Comput., 30 (2008) 1806-1824.

${ }^{23}$ T. Peterson, A note on the convergence of the discontinuous Galerkin method for a scalar hyperbolic equation, SIAM J. Numer. Anal. 28 (1991) 133-140.

${ }^{24}$ W. H. Reed and T. R. Hill, Triangular mesh methods for the neutron transport euqation, Los Alamos Scientific Laboratory Report LA-UR-73-479, 1973.

${ }^{25}$ G. R. Richter, An optimal-order error estimate for the discontinuous Galerkin method, Math. Comp. 50 (1988) 75-88.

${ }^{26}$ P. L. Roe, Approximate Riemann solvers, parameter vectors, and difference schemes, J. Comput. Phys. 43 (1981) 357-372.

${ }^{27} \mathrm{C}$. W. Shu and S. Osher, Efficient implementation of essentially non-oscillatory shock capturing schemes, J. Comput. Phys. 77 (1988) 439-471.

${ }^{28}$ G. I. Taylor and A. E. Green, Mechanism of production of small eddies from large ones, Proc. R. Soc. London A 158 (1937) 499-521.

${ }^{29}$ B. van Leer, Towards the ultimate conservative difference scheme, V. A second order sequel to Godunov's method, J. Comput. Phys. 32 (1979) 101-136.

${ }^{30}$ B. van Leer and S. Nomura, Discontinuous Galerkin for diffusion, AIAA paper (2005) 2005-5108.

${ }^{31}$ B. van Leer, M. Lo and M. van Raalte, A discontinuous Galerkin method for diffusion based on recovery, AIAA paper (2007) 2007-4083.

${ }^{32}$ B. van Leer and M. Lo, Unification of discontinuous Galerkin methods for advection and diffusion, AIAA paper (2009) 2009-400 